\title{
Missing samples reconstruction using an efficient and robust instantaneous frequency estimation algorithm
}

\author{
Sadiq Ali $^{1, *}$ (D), Nabeel Ali Khan ${ }^{2}$ (D) \\ ${ }^{1}$ Department of Electrical Engineering, University of Engineering and Technology, Peshawar, Pakistan \\ ${ }^{2}$ Faculty of Engineering and Information Technology, Foundation University Islamabad, \\ Islamabad, Pakistan
}

Received: 19.08 .2021

Accepted/Published Online: 22.12.2021

Final Version: 31.05.2022

\begin{abstract}
In order to recover missing samples in a nonstationary signal, this paper employs a time-signal analysis and filtering method. The instantaneous frequency of a multicomponent signal is first estimated by employing a robust and computationally efficient method. Then the time-frequency filtering is performed using a dechirping operation to recover missing samples. These steps are repeated until convergence. The proposed method achieves better performance than the state of art methods both in terms of the accuracy of the recovered signal and computational efficiency.
\end{abstract}

Key words: Instantaneous frequency estimation, missing samples, sparse reconstruction, time frequency filtering, AMFM

\section{Introduction}

In many real-life situations, a recorded signal may have missing samples due to the limitations of hardware, multipath effect, and noise [1,2]. Compressive sensing methods have emerged as a powerful tool to recover the missing samples in case of signals that are sparse in a dual-domain [3]. Many nonstationary signals such as wide-band linearly frequency modulated chirps do not have a sparse frequency domain representation but they become sparse in the joint time-frequency (TF) domain. Therefore, for the recovery of nonstationary sparsely sampled signals, time-frequency signal analysis techniques are employed.

Recently a number of TF based sparse reconstruction methods have been developed that include orthogonal matching pursuit based algorithm [4, 5], gradient descent methods $[6,7]$ and TF filtering techniques $[8,9]$. In order to effectively apply the TF signal reconstruction method, it is important to obtain a clean TF representation as missing samples introduce unwanted artifacts. These distortions can be, to a certain extent, mitigated by employing sparsity aware high-resolution TF distributions [1, 10, 10-15].

In a recent study, the Papoulis-Gerchberg approach of recovering stationary signals by employing repeated filtering and insertion of known values was extended for nonstationary signals [9]. The method uses the combination of adaptive directional TF distribution [11] and Viterbi algorithm to estimate the instantaneous frequency (IF) of multicomponent signals $[9,16]$. The IF is then utilized to recover the missing samples by performing TF filtering through dechirping [17]. The known values are then reinserted in the filtered signal and this process is iterated till convergence. The main limitation of the above method is extensive computational cost due to a) computation of adaptive directional TF distribution, b) IF estimation due to the Viterbi algorithm

*Correspondence: sadiqali@uetpeshawar.edu.pk 
[9]. In this study, we overcome the issue of computational cost by employing a robust and efficient IF estimation algorithm that not only reduces the computational cost but also improves the reconstruction performance [18]. Experimental results show the proposed method is better than the recent relevant techniques in the current literature.

The remaining paper is organized as follows: Information of the relevant background for the proposed work are presented in Section 2. The methodology of the proposed method is explained in Section 3 that includes a robust and efficient instantaneous frequency estimation algorithm and the signal reconstruction algorithm. Section 4 contains numerical results results and Section 5 of paper concludes the work.

\section{Background}

The proposed signal reconstruction method is developed by employing the Papoulis-Gerchberg algorithm [19]. Therefore, we briefly review this method in this section. Let us consider $x[n]$ to be a band-pass signal having $M$ nonsparse bins in frequency domain. Similarly, $x_{s}[n]$ is considered to be a signal that is sparsely sampled as: $x_{s}[n]=\operatorname{mask}[n] x[n]$. In the case of missing samples mask $[n]=0$ and mask $[n]=1$ when samples are preserved. The steps of the traditional Papoulis-Gerchberg algorithm are [19]:

- Step 1 Initialization as: $\hat{x}[n]=x_{s}[n]$.

- Step 2 A low pass filter $B\{$.$\} is applied as: \hat{x}[n]=B\{\hat{x}[n]\}$.

- Step 3 Corresponding original values $x_{s}[n]$ are reinserted as: $\hat{x}[n]=\operatorname{mask}[n] x_{s}[n]+(1-\operatorname{mask}[n]) \hat{x}[n]$.

- Step 4 Steps 2 and 3 are iterated till convergence is achieved.

The Papoulis-Gerchberg algorithm converges when the number of observations in signal $x_{s}[n]$ is more than double the number of nonsparse bins. To adopt the Papoulis-Gerchberg algorithm to wide-band multicomponent nonstationary signals [20], the following amplitude modulated-frequency modulated (AM-FM) model is being considered

$$
x[n]=\sum_{k=1}^{M} x_{k}[n]=\sum_{k=1}^{M} a_{k}[n] e^{j \theta_{k}[n]},
$$

where $M$ denotes total number of components, $\theta_{k}[n]$ represents instantaneous phase and $a_{k}[n]$ represents low-pass signal that has a nonoverlapping spectrum with high-pass component $e^{j \theta_{k}[n]}$. It is assumed that $x_{s}[n]$ sparsely sampled version of $x[n]$, i.e. $x_{s}[n]=\operatorname{mask}[n] x[n]$. This means that the components $x_{s_{k}}[n]$ are also sparsely sampled, i.e. $x_{s_{k}}[n]=\operatorname{mask}[n] x_{k}[n]$. So, in order to recover the original signal, it is important to first recover the corresponding signal components.

\section{Proposed method for reconstruction of missing samples}

In the proposed reconstruction algorithm, first the IFs of signal components are estimated and then Papoulis approach of filtering and insertion of low pass filtering is employed in an iterative manner. The key steps of the proposed algorithm are illustrated in Figure 1 and explained in the following sections. 


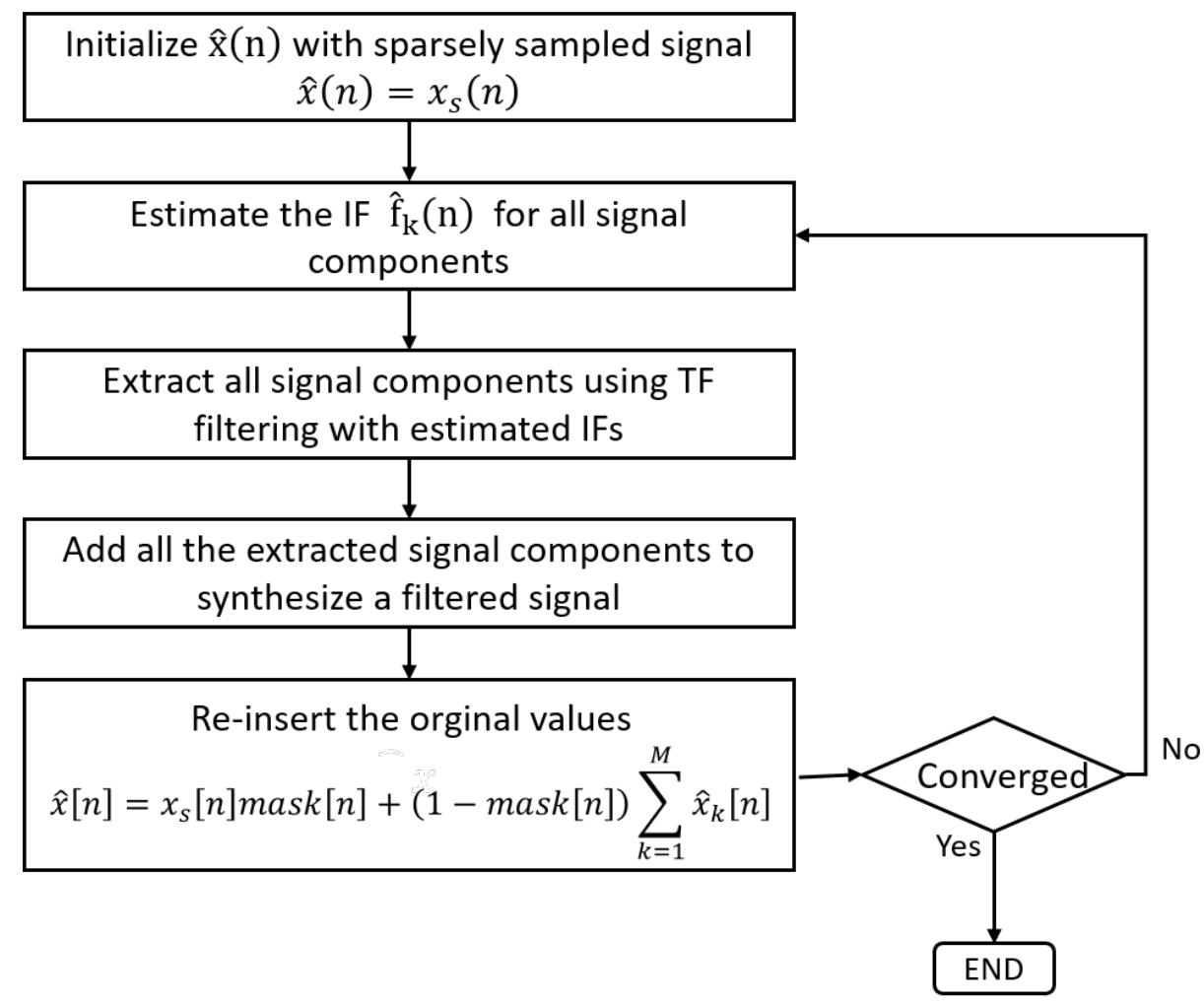

Figure 1. Flow chart of the proposed methodology.

\subsection{Instantaneous frequency estimation}

The algorithm starts by initializing the recovered signal as: $\hat{x}[n]=x_{s}[n]$. Then the process of signal components extraction is performed by using a robust and an efficient IF estimation technique. The following subsections explain the main steps of the component extraction algorithm.

\subsubsection{Finding out highest energy point in TF domain}

For finding the highest energy point, the short-time energy of the signal $\hat{x}[n]$ is found as:

$$
x_{e}[n]=\sum_{k=-\Delta N}^{\Delta N}|\hat{x}[n+k]|^{2},
$$

where $\Delta N$ is a parameter that determines the window width the estimation of signal local energy and is varied to obtain the desired results [18]. Using $x_{e}[n]$, the time-instant where the energy is maximum is found as:

$$
n_{0}=\underset{n}{\operatorname{argmax}} x_{e}[n]
$$




\subsubsection{Finding maximum frequency and rotation order}

Using a precomputed $2 L+1$ Fourier Gaussian windows $w_{\alpha_{l}}(t)$ the signal is windowed. These windows $w_{\alpha_{l}}(t)$ can be expressed as:

$$
w_{\alpha_{l}}(t)=\frac{e^{j \alpha_{l} / 2}}{\sqrt{j \sin \left(\alpha_{l}\right)}} \int_{-\infty}^{\infty} e^{-\frac{\mu^{2}}{2 \sigma^{2}}} e^{j \pi\left(\left(\mu^{2}+t^{2}\right) \cos \left(\alpha_{l}-2 t \mu\right) / \sin \alpha_{l}\right.} d \mu
$$

In order to estimate the location of the peak frequency at time-instant $n_{0}$, we employ $2 L+1$ fractional Fourier Gaussian windows $w_{\alpha_{l}}[n]$ rotated at angles $\alpha_{l}=\frac{l}{L}$, where $l=-L,-L+1, \ldots,-1,0,1, \ldots L$. Similarly, in (4) parameter $\mu$ is integration parameter and $\sigma$ is variance having value 1 . These windows are computed using method described in [21]. Windows $w_{\alpha_{l}}[n]$ are time-shifted at $n_{0}$ and then the Fourier transforms of the windowed signals are computed as:

$$
X_{\alpha_{l}}[k]=\sum_{n=n_{0}-W_{l} / 2}^{n_{0}+W_{l} / 2} x[n] w_{\alpha_{l}}\left[n-n_{0}\right] e^{-\frac{j 2 \pi k n}{N}}
$$

where $W_{l}+1$ is the length of the analysis window and $N$ is the total number of samples in the signal. The location of the maximum frequency $k_{0}$ and rotation order $\alpha_{0}$ of the analysis window at $n_{0}$ are estimated as:

$$
\left(k_{0}, \alpha_{0}\right)=\underset{k, \alpha_{l}}{\arg \max } x_{\alpha_{l}}[k]
$$

Then the IF at time-instant $n_{0}$ for the $i$-th component is given as: $f_{i}\left[n_{0}\right]=k_{0}$.

\subsubsection{Instantaneous frequency estimation}

First we consider the case $n>n_{0}$. Let us initialize $\hat{n}=n_{0}$. Then $\hat{n}$ is incremented as: $\hat{n}=\hat{n}+1$. Through search in a narrow region around $k_{0}-\Delta k \leq k \leq k_{0}+\Delta k$ with the help of windows shifted at $\hat{n}$ and rotated at angles $\alpha_{0}-\Delta \alpha_{0}, \alpha_{0}, \alpha_{0}+\Delta \alpha_{0}$, the IF is estimated at $\hat{n}$ as:

$$
\left(k_{0}, \alpha_{0}\right)=\underset{k, \alpha_{l}}{\arg \max }\left|\sum_{n=\hat{n}-W_{l} / 2}^{\hat{n}+W_{l} / 2} e^{\frac{-j 2 \pi k n}{N}} \hat{x}[n] w_{\alpha_{l}}[n-\hat{n}]\right|,
$$

where parameter $\Delta \alpha_{0}$ is $\frac{1}{2 L+1}$. At time-instant $\hat{n}$ the IF $\hat{f}_{i}[\hat{n}]$ becomes $k_{0}$. The process is repeated till the energy at the peak frequency becomes less than a specified threshold (i.e. a times the energy of the highest energy TF point; in this work $a=0.01$ ).

For the case of $n<n_{0}$ the IF estimation process is similar to the process of IF estimation for the case of $n>n_{0}$.

\subsubsection{Strongest component extraction}

The method described in aforementioned sections estimates the IF of the strongest component. Using this IF information, this component is extracted using the dechirping based TF filter [18]. Once the strongest component is extracted the same procedure is followed to extract the remaining components of the signal. 
Algorithm 1: Summary of the efficient and robust IF estimation method.

1. Initialize $i=1$.

2. Using Eq. (2) the short time energy of a signal at all time-instants computed.

3. The time-instant where energy is maximum is found as: $t_{0}=\underset{t}{\operatorname{argmax}} x_{e}(t)$.

4. Fourier transform is computed using Eqs. ((5) and (4)).

5. The analysis window optimum rotation order and maximum frequency location is found as: $\left(f_{0}, \alpha_{0}\right)=\underset{f, \alpha_{l}}{\arg \max } X_{\alpha_{l}}(f)$.

6. For $k$-th component, find the IF at time-instant $t_{0}$, given as: $f_{i}\left(t_{0}\right)=f_{0}$.

7. For $t>t_{0}$, IF estimation process is:

(a) Initialize $\hat{t}=t_{0}$

(b) Increment $\hat{t}=\hat{t}+1 / f_{s}$

(c) Shift the window at time-instant $\hat{t}$, and estimate the IF by searching around a narrow region around $f_{0}-\Delta f \leq f \leq f_{0}+\Delta f$ using windows rotated at angles $\alpha_{0}-\Delta \alpha_{0} \leq \alpha \leq \alpha_{0}+\Delta \alpha_{0}$ as in Eq. (7).

(d) The IF at time-instant $\hat{t}$ is given as: $f_{i}(\hat{t})=f_{0}$

(e) Repeat from b) until $\hat{t}$ is less than the length of the signal or energy of the peak frequency fall below certain threshold, i.e. 0.1 times the energy of the highest energy TF point.

8. Repeat the same procedure to estimate the IF for $t<t_{0}$ and so the IF of the strongest component is estimated.

9. Using the IF information, the corresponding strongest component is extracted using TF filtering (dechirping).

10. Increment $i=i+1$ and from step 2, the process is repeated to extract the next strongest component.

\subsection{Sparse reconstruction by adopting Papoulis-Gerchberg method}

Using the estimated IF $\hat{f}_{k}[n]$ for $k$-th component, in Section 3.1 the instantaneous phase is estimated as:

$$
\hat{\theta}_{k}[n]=\hat{\theta}_{k}[n-1]+\frac{2 \pi}{N} \hat{f}_{k}[n]
$$

The estimated instantaneous phase $\hat{\theta}_{k}[n]$ is used to get the dechirped signal $x_{d_{k}}[n]$ as:

$$
x_{d_{k}}[n]=\hat{x}_{r}[n] e^{-j \theta_{k}[n]}=a_{r_{k}}[n] e^{j\left(\theta_{k}[n]-\hat{\theta}_{k}[n]\right)}+\sum_{\substack{l=1 \\ k \neq l}}^{M} x_{r_{l}}[n] e^{-j \hat{\theta}_{k}[n]},
$$


where $a_{r_{k}}[n]$ represents the instantaneous amplitude of the $k$-th component of the sparsely sampled signal. Assuming $\hat{\theta}_{k}[n]=\theta_{k}[n]$, the dechirped component becomes

$$
x_{d_{k}}[n]=a_{r_{k}}[n]+\sum_{\substack{l=1 \\ k \neq l}}^{M} x_{r_{l}}[n] e^{-j \theta_{k}[n]} .
$$

The instantaneous amplitudes i.e. $a_{r_{k}}[n]$ of each signal components are low-pass signals that can be recovered using low pass filter $B\{$.$\} as:$

$$
\hat{a}_{k}[n]=B\left\{\hat{x}_{d_{k}}[n]\right\}
$$

In addition to the separation of signal components, the low pass filtering also interpolates the missing samples. The reconstructed signal components become

$$
\hat{x}_{k}[n]=\hat{a}_{k}[n] e^{j \hat{\theta}_{k}[n]}
$$

Finally, the signal is reconstructed by adding all the reconstructed components and reinserting the known samples as:

$$
\hat{x}[n]=x_{s}[n] \operatorname{mask}[n]+(1-\operatorname{mask}[n]) \sum_{k=1}^{M} \hat{x}_{k}[n] .
$$

The process of IF estimation, then signal reconstruction continues until convergence is achieved.

\subsection{Summary of the proposed algorithm}

The key steps of the algorithm are summarized below.

1. Initialize the algorithm as: $\hat{x}[n]=x_{s}[n]$.

2. IFs of all the signal components are estimated using the method given in Section 3.1.

3. Instantaneous phase is estimated as: $\hat{\theta}_{k}[n]=\hat{\theta}_{k}[[n-1]]+\frac{2 \pi}{N} \hat{f}_{k}[n]$.

4. Using Eq. 9, the given signal is dechirped with estimated phase $\hat{\theta}_{k}[n]$.

5. With Eq. 11 instantaneous amplitudes, i.e. $a_{r_{k}}[n]$, are recovered by low-pass filtering.

6. By dechirping the estimated instantaneous amplitudes in step 5, the original signal components are estimated as in Eq. 12.

7. Finally, the signal is reconstructed by adding all the reconstructed components and reinserting the known samples as in Eq. 13.

8. The steps from 2 to 7 are repeated until convergence is achieved. 


\subsection{Computational complexity analysis}

The proposed algorithm iteratively performs the following steps:

- IF of $M$ number of components is estimated.

- TF filtering is performed for each component.

The computational cost of IF estimation step is $O\left(2 M N L \log N+6 \Delta k M W_{L} N\right)$ [22], where $M$ is number of signal components, $N$ is number of samples in the signal, $W_{L}$ is the length of the analysis window, $L$ is the number of quantization levels for estimating the rotation order of fractional Fourier window and $2 \Delta k$ is length of search window to track the strongest TF point. The computational cost of TF filtering is $O(N)$. Let us assume that the algorithm is iterated $P$ times, then the total computational cost of the algorithm becomes $O\left(2 P M N L \log N+6 \Delta k P M W_{L} N+P N\right)$ or simply $O\left(2 P M N L \log N+\Delta k P M W_{L} N\right)$ by ignoring constants and lower order terms.

\section{Results and discussion}

In this section the performance analysis of the proposed algorithm is presented. The numerical results are presented for both synthetic and real signals. For the real signals we consider electroencephalogram (EEG) seizure signals. Details are provided in the following subsections.

\subsection{Synthetic signals}

Let us compare the performance of the proposed method with a Viterbi based signal reconstruction algorithm [23] and orthogonal matching pursuit (OMP) algorithm [24]. We consider two scenarios: 1) randomly missing samples and 2) missing samples in bursts. In each scenario, the signal in use is sampled with $1 \mathrm{~Hz}$, and the duration of the signal is $128 \mathrm{~s}$.

\section{Example 1: Nonlinear frequency modulated chirps}

Let us consider a challenging example of nonlinear frequency modulated chirps that are difficult to analyze using commonly used quadratic TF analysis techniques as such signals cause both inner and outer interference terms. The signal is given as: $z[n]=z_{1}[n]+z_{2}[n]$, where

$$
z_{1}[n]=e^{\left(j 2 \pi\left(0.1 n^{3} / 16384\right)+j 2 \pi(0.05 n)+j 2 \pi\left(0.05 t^{2} / 128\right)\right)}
$$

and

$$
z_{2}[n]=e^{\left(j 2 \pi\left(0.1 n^{3} / 16384\right)+j 2 \pi(0.15 n)+j 2 \pi\left(0.05 n^{2} / 128\right)\right)}
$$

The two components are well separated in the joint TF domain. We randomly remove 48 samples from the signal and then apply the aforementioned signal reconstruction algorithms. The time-domain representation of the original vs. the reconstructed signals (reconstructed with the help of different methods) is shown in Figure 2, whereas the corresponding TF-domain representations are shown in Figure 3. Time-domain representations in Figure 2 illustrate that all methods successfully recover the missing samples, but the best performance is achieved by the proposed method. Similarly, it is also clear from the TF representations given in Figure 3 that the proposed scheme reconstructs the signal having missing samples perfectly. Furthermore, we can see that the TF domain provides clear information compare to time-domain representations, as TF analysis is always 
recommended for the analysis of nonstationary signals. The superior performance of the proposed scheme is further confirmed with the help of mean square error (MSE) between the reconstructed signal and original signal as indicated in Table 1.

(a)

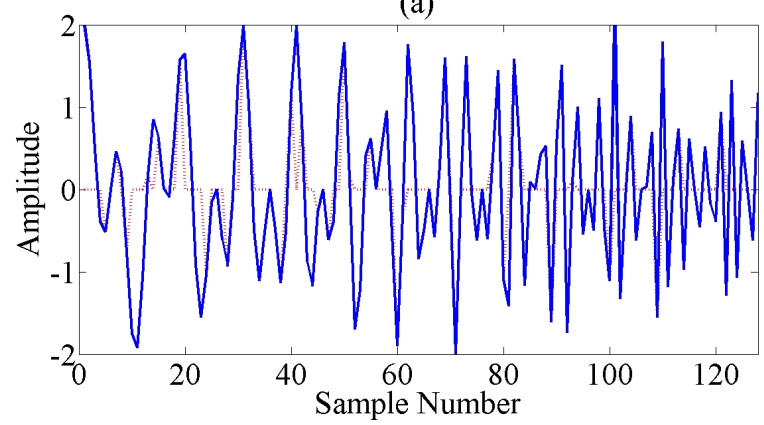

(c)

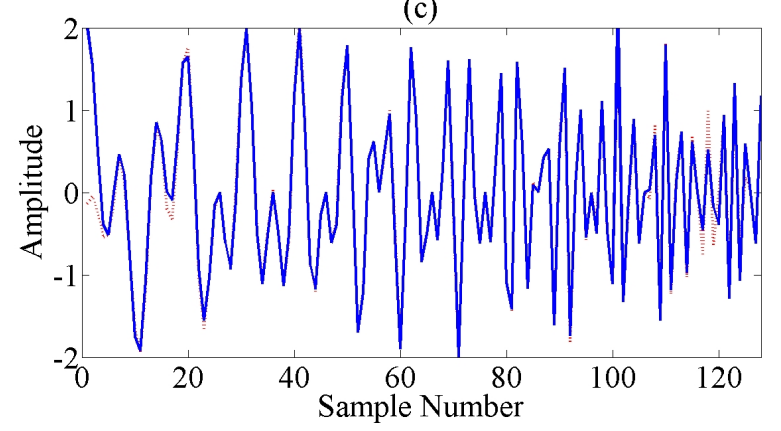

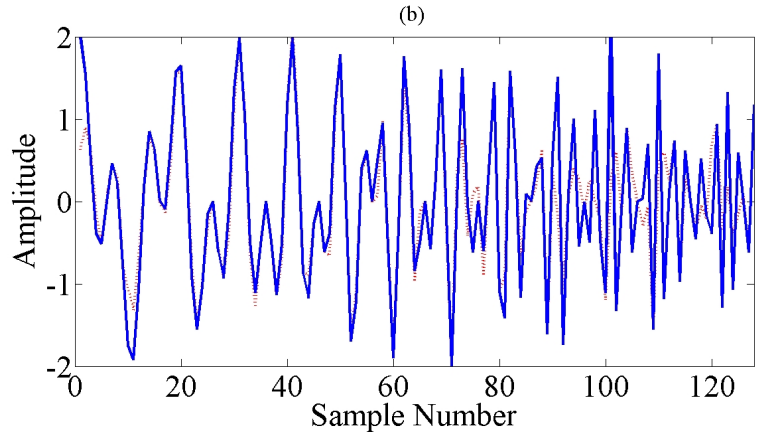

(d)

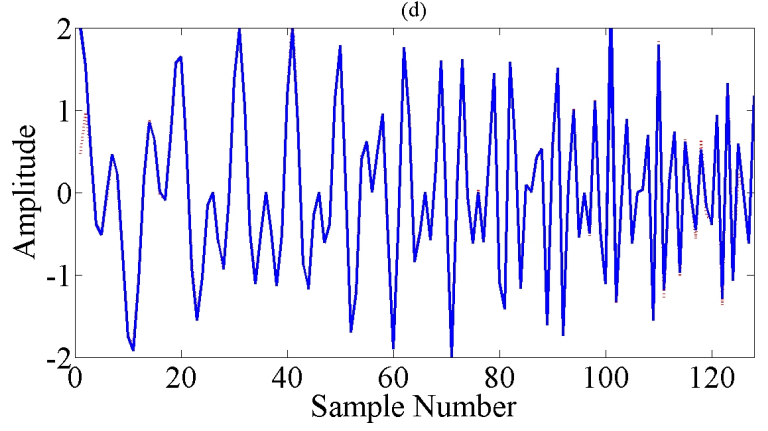

Figure 2. (a) For signal given in the Example 1: (a) the signal with random missing samples(red) vs. the original signal (blue); (b) the reconstructed signal (red) vs. the original signal (blue) using OMP; (c) the reconstructed signal (red) vs. the original signal (blue) using Viterbi algorithm; (d) the reconstructed signal (red) versus the original signal (blue) using the proposed method.

Table 1. For Example 1 signals, the MSE between the reconstructed and original signal.

\begin{tabular}{|l|l|l|}
\hline The proposed method & Viterbi algorithm & OMP variant $[24]$ \\
\hline 0.0354 & 0.1024 & 0.5959 \\
\hline
\end{tabular}

\section{Example 2: Missing samples in gaps}

Let us now consider a scenario when samples are missing from the original signal in gaps. The sparsely sampled signal is now given as:

$$
z[n]=\left\{\begin{array}{l}
0, \quad t \in[10: 30,60: 80,90: 130] \\
z[n], \quad \text { otherwise }
\end{array}\right.
$$

Figures 4 and 5 illustrate the time-domain and corresponding time-frequency domain representations of the original signal, sparsely sampled signal and reconstructed signals obtained using the proposed methodology. These results indicate that the proposed scheme perform better than the other state of the art methods from recent literature. Similarly, the mean square error in Table 2 also illustrates that the proposed method achieves the best accuracy. 
(a)

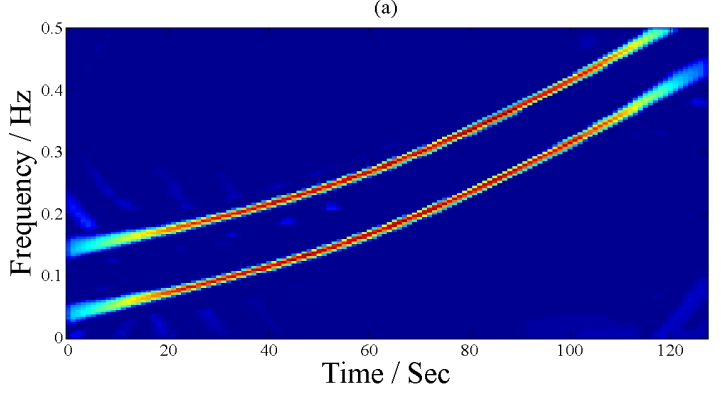

(c)

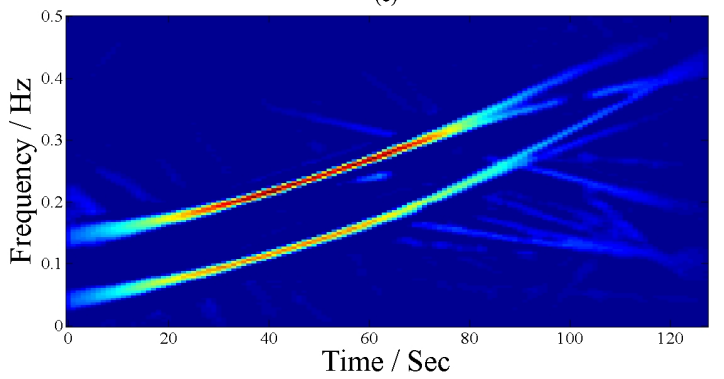

(a)

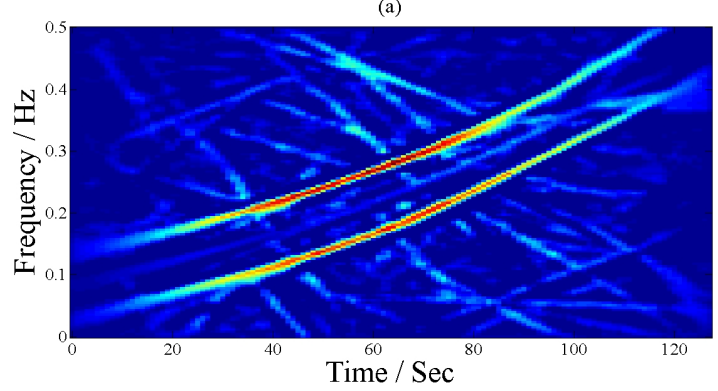

(d)

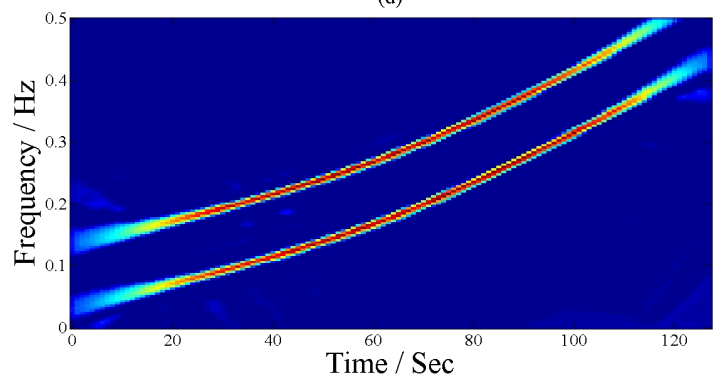

(e)

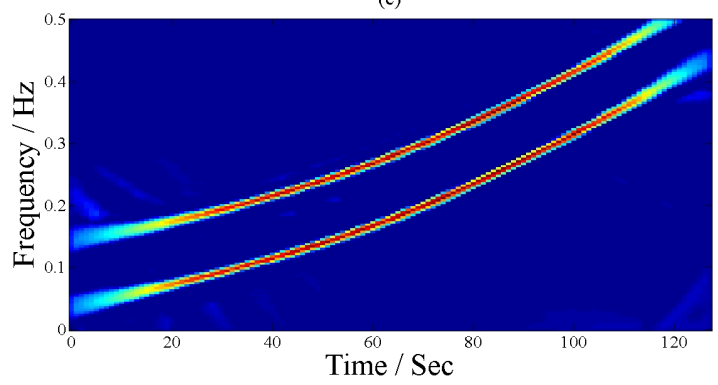

Figure 3. (a)TF representation of the original signal; (b)TF representation of signal with missing samples; (c) TF representation of the reconstructed signal obtained using OMP; (d) TF representation of the reconstructed signal obtained using Viterbi algorithm; (e) TF representation of the reconstructed signal using the proposed method.

Table 2. Example 2 signal: The MSE between the reconstructed signal and original signal.

\begin{tabular}{|l|l|l|}
\hline Viterbi method [9] & Proposed method (last iteration) & OMP variant [24] \\
\hline 0.0636 & 0.0304 & 1.0698 \\
\hline
\end{tabular}

\section{Example 3: Sparsely sampled signal with intersecting components}

Let us now consider an example of sparsely sampled signal with intersecting components. The signal is defined as: $z[n]=z_{1}[n]+z_{2}[n]$, where

$$
z_{1}[n]=e^{\left(j 2 \pi\left(0.1 n^{3} / 16384\right)+j 2 \pi(0.05 n)+j 2 \pi\left(0.05 n^{2} / 128\right)\right)}
$$

and

$$
z_{2}[n]=e^{\left(-j 2 \pi\left(0.1 n^{3} / 16384\right)+j 2 \pi(0.45 n)-j 2 \pi\left(0.05 n^{2} / 128\right)\right)} .
$$



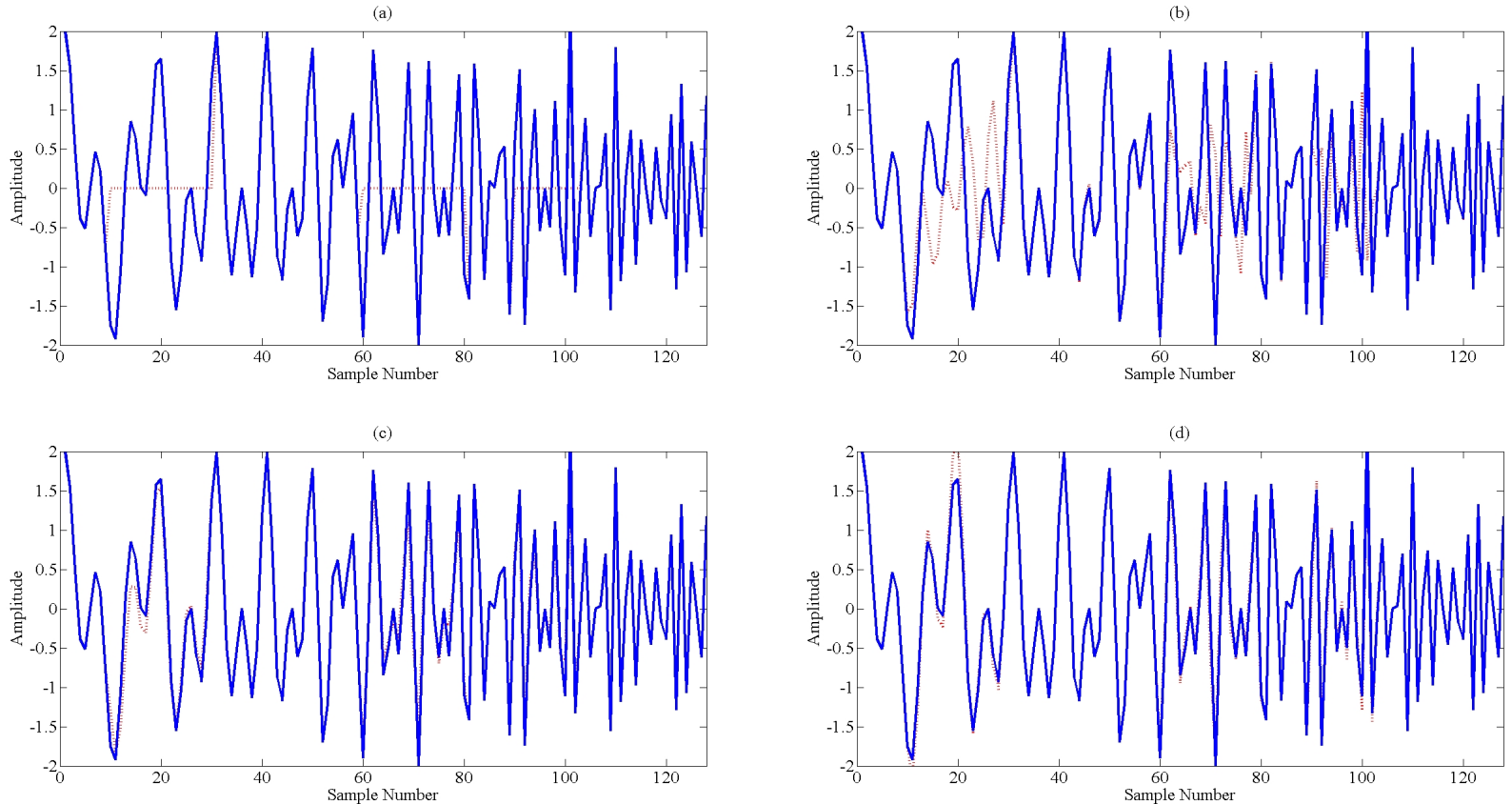

Figure 4. (a) For Example 2 signal: the original signal (blue) vs. the signal with missing samples in random (red) and the reconstructed signal (red) vs. the original signal (blue) based on the (b) OMP (c) Viterbi algorithm; (d) the proposed method.

We remove samples from the signal is gaps, the new signal is given as:

$$
z[n]=\left\{\begin{array}{l}
0, \quad t \in[11: 30,60: 75,90: 101] \\
z[n], \quad \text { otherwise. }
\end{array}\right.
$$

Time-domain representation and corresponding TF domain representation of the original signal, signal with missing samples, and reconstructed signal are given in Figures 6 and 7, respectively. These results clearly indicate and confirm that in such scenario the proposed algorithm perfectly reconstruct the signal. Experimental results for mean square error obtained in Table 3 indicate that the proposed method outperforms the other state of the art methods from the recent literature.

Table 3. The MSE between the reconstructed and the original signal given in Example 3.

\begin{tabular}{|l|l|l|}
\hline Viterbi & The proposed method & OMP variant $[24]$ \\
\hline 0.2349 & 0.1410 & 0.9923 \\
\hline
\end{tabular}

\section{EEG seizure signals with missing samples}

Let us now consider an example of EEG seizure signal obtained from [25]. The signal is sampled at $16 \mathrm{~Hz}$ and is of 8-s duration. For the interested readers more details about the DATA could be found from [25]. The TF representation of the signal is give in Figure 8a. We remove 56 samples from the signal as:

$$
z[n]=\left\{\begin{array}{l}
0, \quad t \in[10: 25,40: 54,70: 84,100: 109] \\
z[n], \quad \text { otherwise. }
\end{array}\right.
$$


(b)

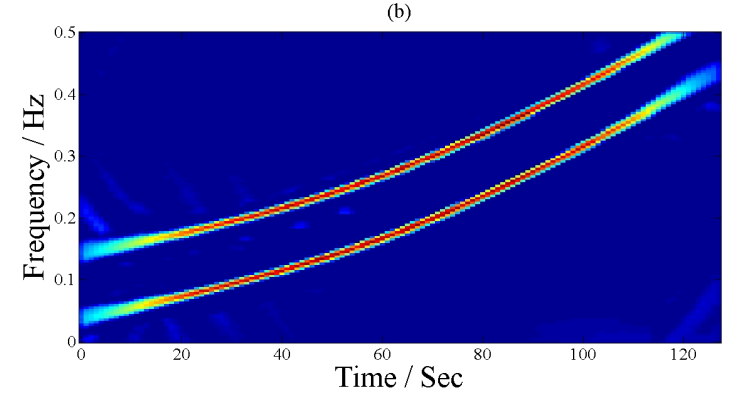

(c)

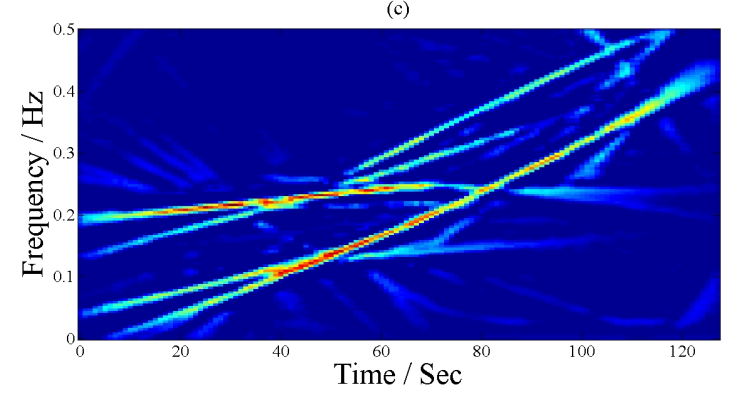

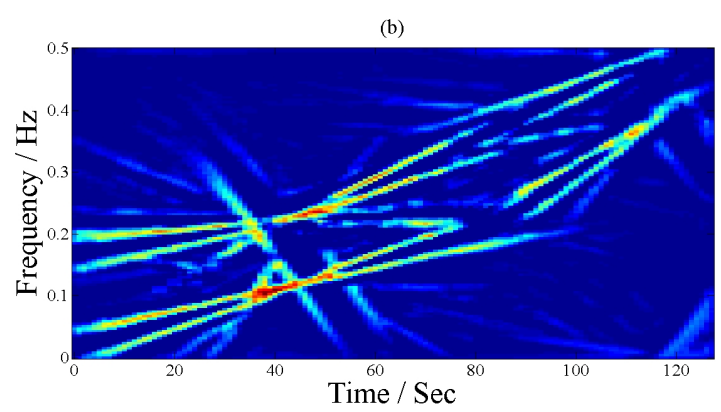

(d)

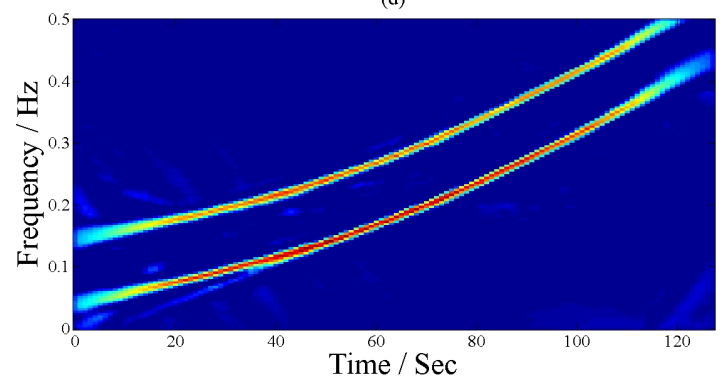

(e)

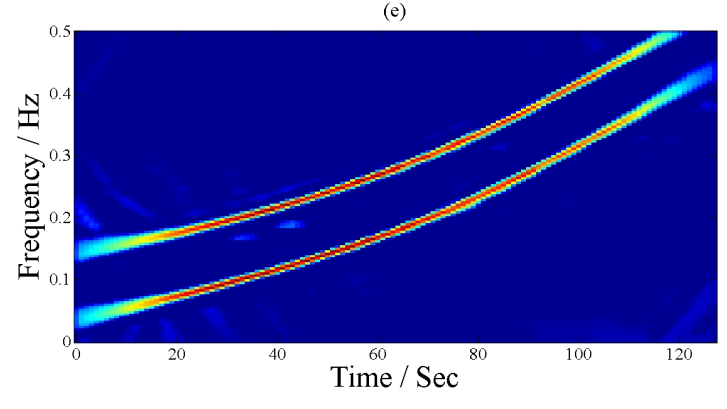

Figure 5. TF representation (a) original signal; (b) the signal with missing samples; (c) the reconstructed signal obtained using OMP; (d) the reconstructed signal obtained using Viterbi algorithm; (e) the reconstructed signal using the proposed method.

The TF representation of the sparsely sampled signal is given in Figure 8b. The signal is reconstructed by the applying the proposed method, Viterbi algorithm and OMP algorithm. The TF representation of the reconstructed signal using OMP is shown in Figure 8c, reconstructed signal using Viterbi algorithm is shown in Figure 8d and reconstructed signal using the proposed method is shown Figure 8e. These representations indicate that the best performance is obtained by the proposed method. This is also confirmed from Table 4 that proposed method achieves the minimum MSE between the original signal and the reconstructed signal.

Table 4. The MSE between the reconstructed and the original signal given in Example 3.

\begin{tabular}{|l|l|l|}
\hline Viterbi & The proposed method & OMP variant [24] \\
\hline 0.0012 & $7.2089 e-04$ & 0.0016 \\
\hline
\end{tabular}


(a)

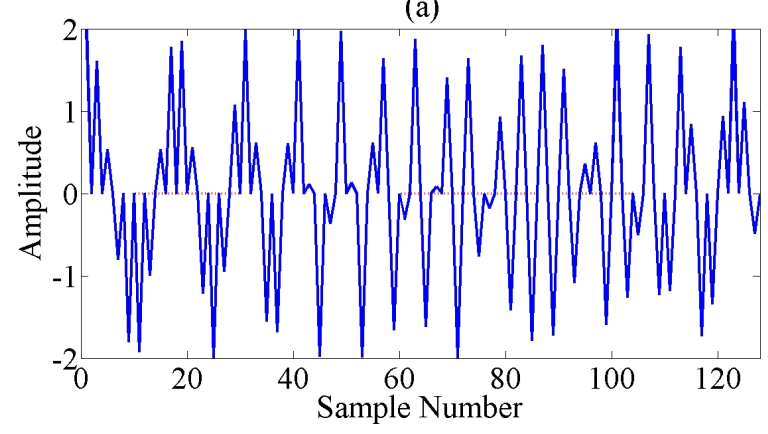

(c)

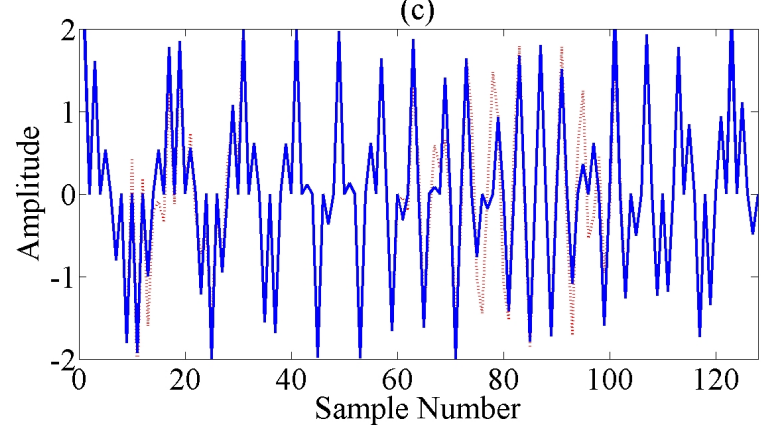

(b)

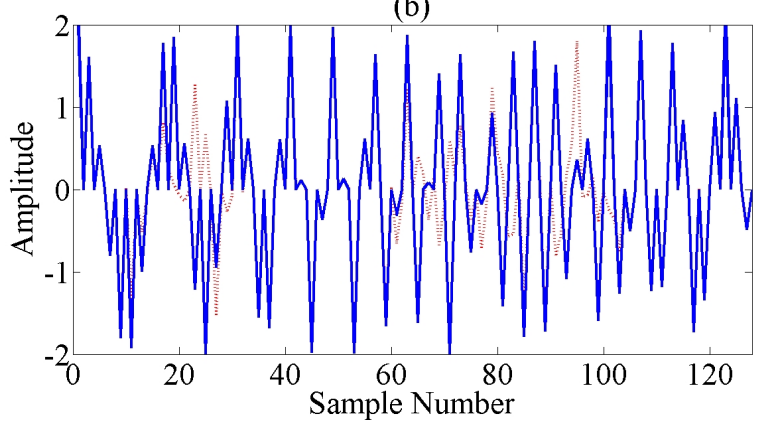

(d)

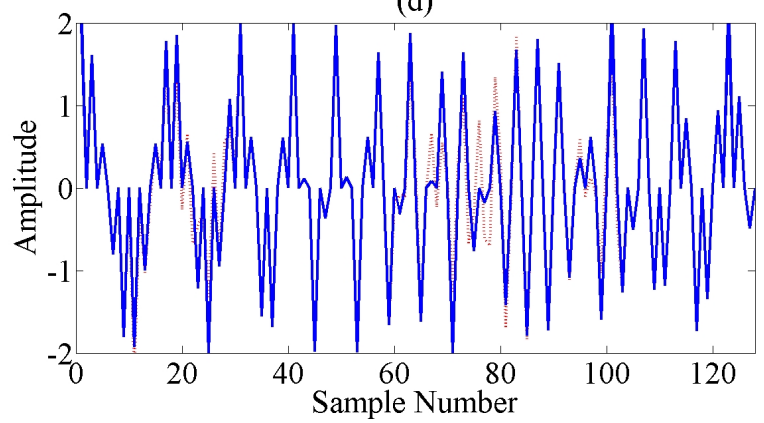

Figure 6. (a) The original signal (blue) vs. the signal with missing samples in random (red), corresponding to the signal given in Example 3; and the reconstructed signal (red) vs. the original signal (blue) based on the (b) proposed method; (c) Viterbi algorithm and (d) OMP variant.

\subsection{Interpretation of results}

Experimental results indicate that the proposed reconstruction method achieves better performance than both OMP and Viterbi based algorithms. The OMP based method fails to achieve good performance when signals are missing in gaps. The performance of both the proposed method and Viterbi based algorithm depends on the accurate estimation of the IF curve, particularly in the case of components that are very close in the TF domain. The IF estimation method employed in this study achieves better performance than the Viterbi based method, as also demonstrated in earlier studies [18], thus leading to improved performance in sparse reconstruction. The proposed algorithm not only achieves better reconstruction performance but it is also computationally efficient, i.e. its computational cost is $O\left(2 P M N L \log N+\Delta k P M W_{L} N\right)$, whereas the computational cost of the Viterbi based algorithm is $O\left(N^{3}\right)$ [9]. The efficacy of the proposed method for the reconstruction of missing samples in EEG seizure signals indicates that the sparse reconstruction methods can be used for data compression and/or removal of artifacts (that can be interpreted as missing samples).

\section{Conclusion}

A computationally efficient and robust signal reconstruction method has been developed that is applicable for both signals with randomly missing samples and signals with samples missing in gaps. The proposed method first estimates the instantaneous frequencies (IFs) of sparsely sampled signals using a robust and efficient algorithm. The estimated IFs are then used to recover the missing samples and this process is iterated till convergence. Experimental results indicate that the proposed method outperforms the state of the art both in terms of the accuracy of the reconstruction and computational cost requirements. 


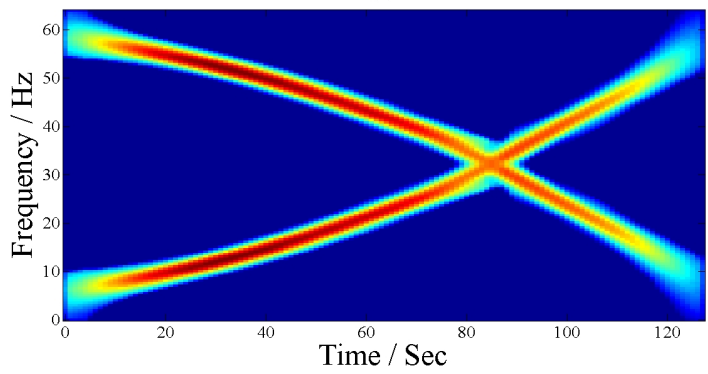

(c)

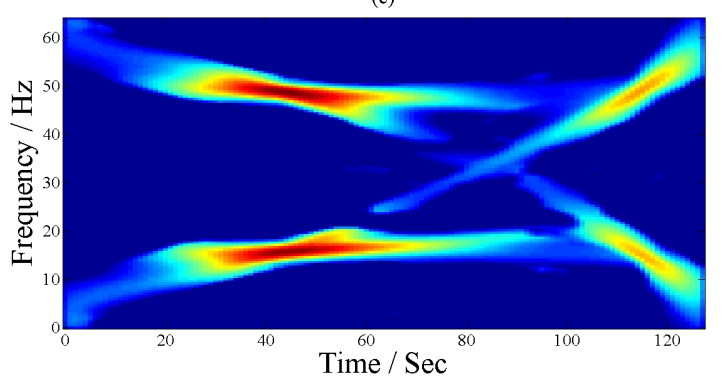

(b)

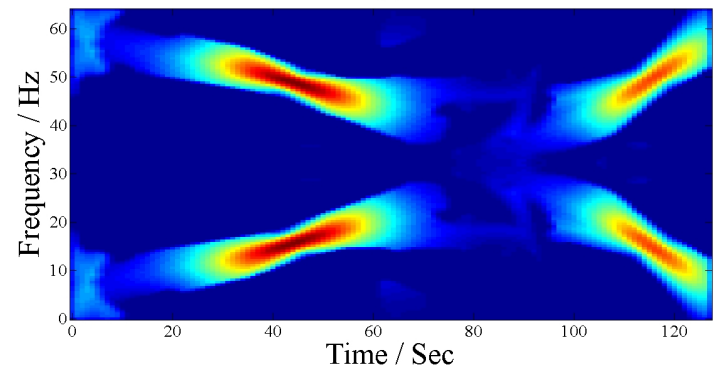

(d)

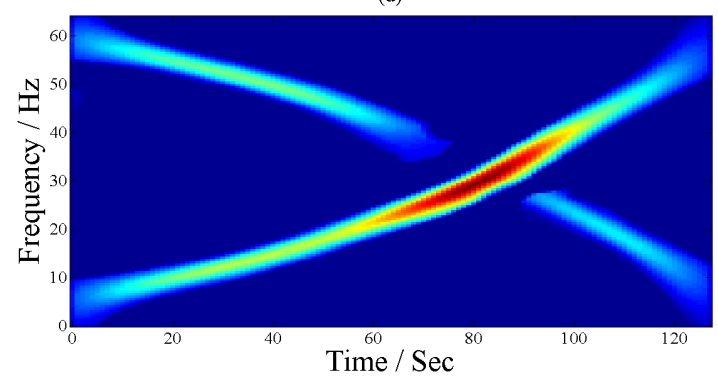

(e)

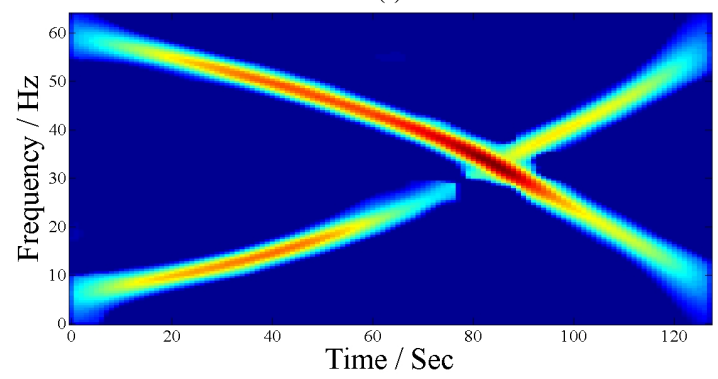

Figure 7. (a) TF representation of original signal; (b) TF representation of the signal with missing samples; (c) TF representation of the reconstructed signal obtained using OMP; (d) TF representation of the reconstructed signal obtained using Viterbi algorithm; (e) TF representation of the reconstructed signal using the proposed method.

\section{Acknowledgment}

The research was supported by Foundation University Islamabad (grant no. FUI/ORIC/IFP-Grant\#78).

\section{Contribution of authors}

N.A. Khan and S. Ali proposed the idea, N.A. Khan and S. Ali did the experiments, S. Ali interpreted the results, S. Ali wrote the paper and N.A. Khan checked the final writings. 
(a)

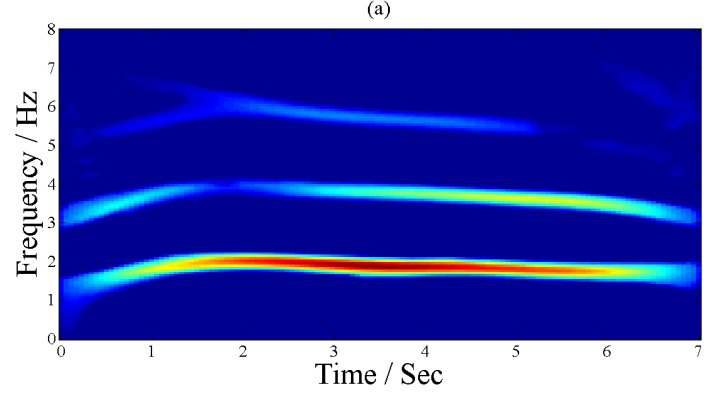

(c)

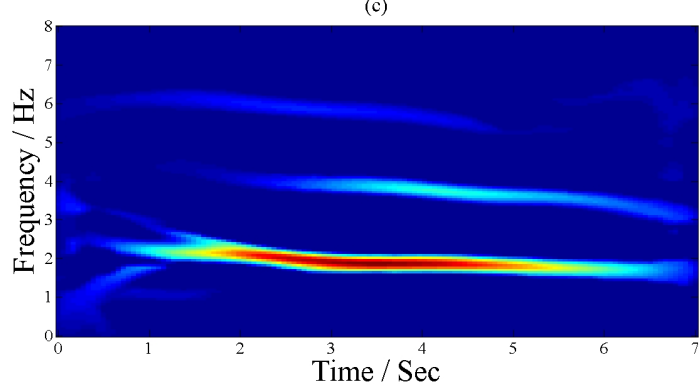

(b)

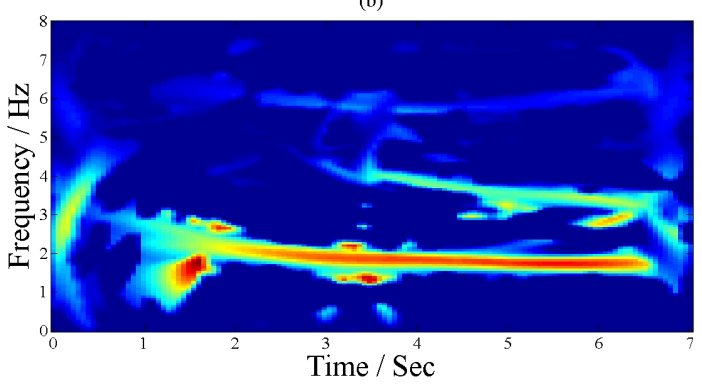

(d)

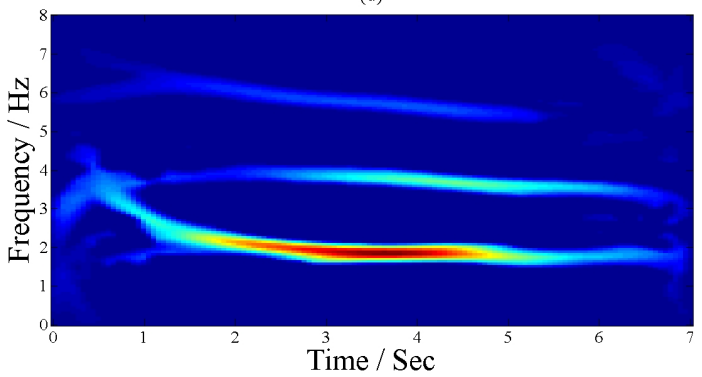

(e)

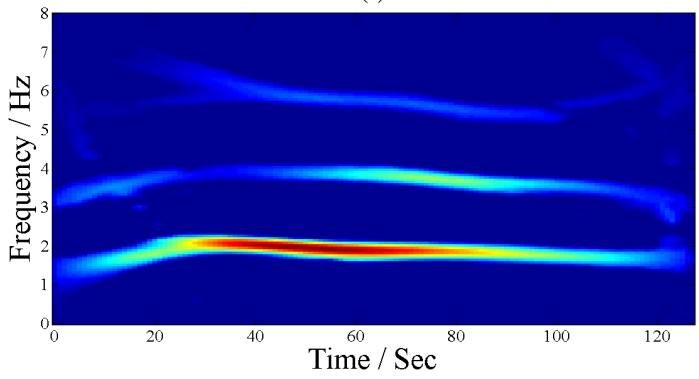

Figure 8. (a) TF representation of original signal; (b) TF representation of the signal with missing samples; (c) TF representation of the reconstructed signal obtained using OMP; (d) TF representation of the reconstructed signal obtained using Viterbi algorithm; (e) TF representation of the reconstructed signal using the proposed method.

\section{References}

[1] Amin VS, Zhang YD, Himed B. Sparsity-based time-frequency representation of FM signals with burst missing samples. Signal Processing. 2019; 155:25-43.

[2] Amin MG, Borio D, Zhang Y, Galleani L. Time-Frequency Analysis for GNSSs: From interference mitigation to system monitoring [Journal Article]. IEEE Signal Processing Magazine. 2017;34 (5):85-95.

[3] Stanković L, Sejdić E, Stanković S, Daković M, Orović I. A Tutorial on Sparse Signal Reconstruction and Its Applications in Signal Processing. Circuits, Systems, and Signal Processing. 2018

[4] Needell D, Tropp JA. CoSaMP: Iterative signal recovery from incomplete and inaccurate samples. Applied and Computational Harmonic Analysis. 2009;26 (3):301 321.

[5] Tropp JA. Greed is good: algorithmic results for sparse approximation. IEEE Transactions on Information Theory. 2004;50 (10):2231-42.

[6] Stanković L, Dakovic M, Vujovic S. Adaptive variable step algorithm for missing samples recovery in sparse signals. IET Signal Processing. $2014 ; 8$ (3):246-56. 
ALI and KHAN/Turk J Elec Eng \& Comp Sci

[7] Blumensath T, Davies ME. Gradient Pursuits. IEEE Transactions on Signal Processing. 2008 ;56 (6):2370-82.

[8] Khan NA, Mohammadi M. Reconstruction of Non-stationary Signals with Missing Samples Using Time-frequency Filtering. Circuits, Systems, and Signal Processing. 2018 ;37 (8):3175-90.

[9] Khan NA, Mohammadi M, Stankovic I. Sparse reconstruction based on iterative TF domain filtering and Viterbi based IF estimation algorithm. Signal Processing. 2020; 166:107260.

[10] Jokanovic B, Amin M. Reduced Interference Sparse Time-Frequency Distributions for Compressed Observations. IEEE Transactions on Signal Processing. 2015;63 (24):6698-709.

[11] Khan NA, Ali S. Sparsity-Aware Adaptive Directional Time-Frequency Distribution for Source Localization. Circuits, Systems, and Signal Processing. 2018 ;37 (3):1223-42.

[12] Amin MG, Jokanovic B, Zhang YD, Ahmad F. A sparsity-perspective to quadratic time-frequency distributions. Digital Signal Processing. 2015;46:175 190.

[13] Liu S, Zhang YD, Shan T, Tao R. Structure-aware Bayesian compressive sensing for frequency-hopping spectrum estimation with missing observations. IEEE Transactions on Signal Processing. 2018;66 (8):2153-66.

[14] Zhang S, Zhang YD. Robust Time - Frequency Analysis of Multiple FM Signals With Burst Missing Samples. IEEE Signal Processing Letters. 2019;26 (8):1172-6.

[15] Orović I, Stanković S, Thayaparan T. Time-frequency-based instantaneous frequency estimation of sparse signals from incomplete set of samples. IET Signal Processing. 2014;8 (3):239-45.

[16] Khan NA, Mohammadi M, Djurović I. A Modified Viterbi Algorithm-Based IF Estimation Algorithm for Adaptive Directional Time-Frequency Distributions. Circuits, Systems, and Signal Processing. 2019 ;38 (5):2227-44.

[17] Yang Y, Dong X, Peng Z, Zhang W, Meng G. Component extraction for non-stationary multi-component signal using parameterized de-chirping and band-pass filter. IEEE Signal Processing Letters. 2014;22 (9):1373-7.

[18] Khan NA, Ali S. A robust and efficient instantaneous frequency estimator of multi-component signals with intersecting time-frequency signatures. Signal Processing 2020; 177:107728.

[19] Papoulis A. A new algorithm in spectral analysis and band-limited extrapolation. IEEE Transactions on Circuits and Systems. 1975;22 (9):735-42.

[20] Daneshvar M, Salehi P. Optimization of multicomponent signals entered to the system using estimation of instantaneous frequency. Journal of Vibration and Control. doi:10.1177/1077546320986381

[21] Bultheel A, Martinez Sulbaran HE. Computation of the fractional Fourier transform. Applied and Computational Harmonic Analysis. 2004;16 (3):182-202.

[22] Khan NA, Ali S. An efficient IF estimation algorithm for both mono-and multi-sensor recordings. Signal, Image and Video Processing. 2021;15:1687-1693.

[23] Stanković L, Dakovic M, Vujovic S. Adaptive variable step algorithm for missing samples recovery in sparse signals. IET Signal Processing. 2014;8 (3):246-56.

[24] Stanković I, Ioana C, Dakovic M. On the reconstruction of nonsparse time-frequency signals with sparsity constraint from a reduced set of samples. Signal Processing. 2018;142:480-4.

[25] Boashash B, Khan NA, Ben-Jabeur T. Time-frequency features for pattern recognition using high-resolution TFDs: A tutorial review. Digital Signal Processing. 2015;40:1 30. 